Meta

Journal des tradlucteurs

Translators' Journal

\title{
Lexique anglais-français de la régate
}

\section{Pierre Biron}

Volume 24, numéro 4, décembre 1979

URI : https://id.erudit.org/iderudit/002082ar

DOI : https://doi.org/10.7202/002082ar

Aller au sommaire du numéro

Éditeur(s)

Les Presses de l'Université de Montréal

ISSN

0026-0452 (imprimé)

1492-1421 (numérique)

Découvrir la revue

Citer cet article

Biron, P. (1979). Lexique anglais-français de la régate. Meta, 24(4), 465-471.

https://doi.org/10.7202/002082ar d'utilisation que vous pouvez consulter en ligne.

https://apropos.erudit.org/fr/usagers/politique-dutilisation/ 


\section{LEXIQUE ANGLAIS-FRANÇAIS DE LA RÉGATE}

ABANDONMENT (of a race by the race committee, after the starting signal) interruption ; invalidation

ABANDONMENT AND RE-SAIL SIGNAL (Code flag $N$ over $X$ and three guns) signal d'interruption et de nouveau départ; signal d'invalidation et de nouveau départ

ABANDONMENT SIGNAL (Code flag $\mathrm{N}$ and three guns) signal d'interruption ; signal d'invalidation

ABANDONED RACE (by the race committee) course invalidée; course interrompue

AGROUND échoué

ALTER COURSE, TO modifier sa route

ANCHORED mouillé

ANSWERING PENDANT (Postponement signal) aperçu

APPROVED CLASS RULES règlements approuvés de série

BASIC RULE règle fondamentale

BEAR AWAY, TO laisser porter

BEAT TO WINDMARK, TO louvoyer ; courir des bords

BEYOND HEAD TO WIND au-delà de la position debout au vent

BLUE SHAPE (Finishing signal) voyant bleu

BOATBUILDER'S MARK marque de fabrique du constructeur

BOOMED HEADSAIL foc bômé

CALL ATTENTION TO SIGNALS, TO attirer l'attention sur les signaux

CANCELLATION annulation 
CANCELLATION SIGNAL (N over first substitute and three guns) signal d'annulation

CAPSIZED chaviré

CHANGE SAILS, TO changer de voiles

CHANGE TACK, TO changer de bord

CLASS série ; classe

CLASS EMBLEM emblème de série

CLASS OWNERS' ASSOCIATION association des propriétaires de la classe

CLASS RECALL rappel d'une série

CLASS RULES règles de série

CLEAR AHEAD en route libre devant

CLEAR ASTERN en route libre derrière

CLOSE-HAULED COURSE route au près; route au plus près

COME WITHIN HAIL! venez à portée de voix !

COMMERCIAL SPONSORSHIP OF A RACE patronage commercial d'une course

COURSE parcours

COURSE SIGNAL signal de parcours

CROSS THE LINE, TO franchir, couper la ligne

CURTAILED LUFF lof écourté

DECISION OF PROTESTS jugement des réclamations

DISQUALIFIED disqualifié

DISTINGUISHED NUMBER numéro distinctif

DRAG, TO chasser

ESTABLISH AN OVERLAP, TO établir un engagement

ESTABLISH AND MAINTAIN AN OVERLAP, TO établir et maintenir un engagement

FAIR SAILING navigation correcte; navigation loyale

FINISH, TO finir

FINISH A SHORTENED COURSE, TO finir un parcours réduit

FINISHING LINE ligne d'arrivée

FINISHING MARK marque d'arrivée

FINISHING SIGNAL signal de fin de course

FIRST SUBSTITUTE (General recall signal) premier substitut

FOLLOW ME! suivez-moi !

FORESTAY étai ; étai avant 
FORM OF ENTRY formule d'inscription

FREE LEG bord portant ; partie portante ; bord allures-portantes

GENERAL RECALL rappel général

GIVE ROOM, TO donner de la place

GYBE, TO empanner

GYBE empannage

GYBE MARK marque sous le vent

GYBING empannage

GYBING YACHT yacht empannant

HAIL, TO héler; appeler à la voix; appeler

HAIL A YACHT, TO héler un yacht

HAILED YACHT yacht hélé

HAILING appel à la voix; appel

HAILING FOR ROOM TO TACK AT OBSTRUCTIONS appel pour demander la place de virer de bord aux obstacles

HAILING YACHT yacht qui a hélé

HEARING OF PROTESTS examen des réclamations

I TACK! je vire !

INDIVIDUAL RECALL rappel individuel

INFRINGING YACHT yacht en infraction

INSIDE OVERLAP engagement à l'intérieur

INSIDE YACHT yacht à l'intérieur

INTERNATIONAL CODE FLAG pavillon du code international

JIB TACK amure de foc; point d'amure de foc

JIBE, TO empanner

JIBE empannage

JIBING empannage

KEEP CLEAR, TO s'écarter

LAY LINE route normale

LEADING YACHT yacht de tête; yacht en tête

LEAVE ALL MARKS TO PORT, TO laisser toutes les marques à bâbord

LEAVE ALL MARKS TO STARBOARD, TO laisser toutes les marques à tribord

LEEWARD SIDE côté sous le vent

LEG OF A COURSE partie d'un parcours

LIFE-JACKET SIGNAL signal des gilets de sauvetage 
LIFE-SAVING EQUIPMENT engins de sauvetage

LUFF, TO lofer

LUFF ABOVE A CLOSE-HAULED COURSE, TO lofer au-delà du près serré

LUFFING lof

LUFFING RIGHTS droits de lof

MAN OVERBOARD homme à la mer

MARK marque

MARK SIGNAL pavillon de marque

MAST ABEAM ! (Mainmast of leeward yacht abeam of helmsman of windward yacht) mât par le travers !

MEASUREMENT CERTIFICATE certificat de jauge

MEASUREMENT RULE règle de jauge ; jauge

MISSING MARK marque manquante

NATIONAL LETTER lettre de nationalité

NOTICE OF RACE programme de course

OBLIGATIONS obligations

OBSTRUCTION obstacle

OFFWIND LEG partie (de parcours) portante ; bord portant

OLYMPIC SCORING SYSTEM mode olympique de décompte des points

ON A TACK sur un bord

ONUS OF PROOF charge de la preuve

OOCHING ooching; saccades

OPPOSITE TACKS bords opposés ; amures opposées

OUTRIGGER tangon débordeur

OUTSIDE OVERLAP engagement à l'extérieur

OUTSIDE YACHT yacht à l'extérieur

OVERLAP (between two yachts) engagement

OVERLAP FROM CLEAR ASTERN engagement par un yacht rattrapant

OVERLAP LIMITATIONS limitations de l'engagement

OVERLAPPING YACHT yacht engagé

OWNER STEERING ANOTHER BOAT propriétaire barrant un autre yacht PASS A MARK, TO contourner, doubler une marque

PORT ROUNDING OF A MARK virement d'une marque laissée à bâbord PORT TACK, ON bâbord-amures 
POSTPONE A RACE, TO (before it starts) remettre une course ; retarder une course

POSTPONED RACE (by the race committee) course retardée ; course remise PREPARATORY SIGNAL (Blue shape, 5 minutes, one gun) signal d'avertissement ; signal préparatoire

PROPER COURSE (after the start) route normale

PROTEST SIGNAL pavillon de réclamation

PROTESTOR réclamant

PUMPING pumping; pompage

RACE COMMITTEE comité de course

RACING YACHT yacht en course

RANKING AS A STARTER considéré comme partant

RECALL rappel

RE-ROUND A MARK, TO contourner à nouveau une marque

RESAIL A RACE, TO recourir une course

RESAILED RACE course recourue

RESPONDING TO HAILING réponse à l'appel

RETURN TO START, TO revenir prendre le départ

RIGHT OF WAY droit de priorité ; priorité

RIGHT OF WAY RULES règles de route

RIGHT-OF-WAY YACHT yacht prioritaire

RIGHTS droits

ROCKING rocking; roulis

ROUND A MARK, TO doubler, contourner une marque

ROUND A MARK TO STARBOARD, TO virer une marque laissée à tribord

ROUND THE ENDS STARTING RULE règle du retour par l'extérieur

RULE INFRINGEMENT infraction aux règlements

SAIL ABOVE A PROPER COURSE, TO lofer au-delà de la route normale

SAIL ABOVE CLOSE-HAULED, TO lofer au-delà du près, du plus près

SAIL ABOVE THE COURSE TO A MARK, TO faire un près au-dessus d'une marque

SAIL BELOW A PROPER COURSE, TO faire une route plus arrivée que la route normale ; laisser porter au-delà de la route normale

SAIL CLOSE TO THE WIND, TO serrer le vent

SAIL NUMBER numéro de voilure 
SAIL THE COURSE, TO effectuer le parcours

SAILING INSTRUCTIONS instructions de course

SAILMAKER'S MARK marque de fabrique du voilier, du maitre-voilier

SAME TACK même amure ; même bord

SCANTLING RULE règle d'échantillonnage

SCORING SYSTEM mode de décompte des points

SET THE SAILS, TO établir les voiles

SHAPE voyant

SHEET SAILS ON A SPAR, TO border les voiles sur un espar

SHEET THE SAILS, TO border les voiles

SHIFTING BALLAST déplacement du lest

SHIGHTING ABEAM FROM HIS NORMAL STATION regardant par le travers de sa place normale

SHIPPING BALLAST embarquement de lest

SHORTEN COURSE SIGNAL signal de réduction de parcours

SKIPPERS' MEETING réunion des skippers

SPINNAKER BOOM tangon de spi, de spinnaker

SPONSOR A RACE, TO (said of a club, etc.) organiser une course

STARBOARD TACK, ON tribord-amures

START, TO prendre le départ

START départ

STARTING AREA zone de départ

STARTING LINE ligne de départ

STARTING SIGNAL (Red shape, 0 minute, one gun) signal de départ

SURROUNDED BY NAVIGABLE WATER entouré d'eau navigable

TACK (Manœuver) virement de bord

TACK, TO virer de bord

TACKING YACHT yacht virant de bord

TAKE IN A SAIL, TO amener une voile

TEAM RACING course d'équipe, par équipe

TEAM RACING RULES règlements de course d'équipe

TIE eX-æquo

TIME LIMIT FOR FINISHING temps limite pour finir

TIME OF START heure du départ

TIME THE START OF A YACHT, TO chronométrer le départ d'un yacht 
En poussant la recherche un peu plus loin dans le but de relever des occurrences de ces expressions dans des ouvrages spécialisés, on en découvre également des variantes où, d'ailleurs, la langue ne gagne rien en concision ni en maniabilité :

- $\quad$ sens des aiguilles d'une montre

sens opposé aux aiguilles d'une montre 16,18

- $\quad$ sens opposé des aiguilles d'une montre 23

- $\quad$ sens des aiguilles d'une montre

sens inverse des aiguilles d'une montre $21,23,26$

- dans le sens des aiguilles d'une montre

contre le sens des aiguilles d'une montre 25

- $\quad$ sens du mouvement des aiguilles d'une montre

sens inverse du mouvement des aiguilles d'une montre 17

- $\quad$ sens des aiguilles d'une montre

sens inverse de celui des aiguilles d'une montre 11

Il est intéressant de noter à ce sujet que ces formulations sont tantôt des équivalents proposés directement pour les termes anglais clockwise et counter clockwise ou anti-clockwise, tantôt des explications ou des définitions d'expressions françaises moins courantes appartenant à des langues de spécialités (voir plus loin). D'ailleurs, le nombre même de ces variantes peut constituer une preuve de cette fonction explicative ou définitoire.

Par association d'idées, passons des aiguilles de montre à l'horloge au fil des exemples suivants :

- $\quad$ rotation sens d'horloge

«... La fermeture s'obtient par rotation sens d'horloge de l'organe de commande ou de son extrémité de manœuvre $\$ 2$

- $\quad$ rotation en sens d'horloge 5

- $\quad$ tournant dans le sens d'horloge 27

- $\quad$ sens inverse d'horloge 18

Toujours dans l'imagerie horlogère, le sens d'horloge nous conduit logiquement aux adjectifs horaire et antihoraire, en électricité et appareils électriques, dans des expressions comme :

- $\quad$ sens horaire

sens antihoraire 10

- attaque horaire (clockwise operation)

attaque antihoraire (counter-clockwise operation) 9

- $\quad$ rotation horaire (clockwise operation)

rotation antihoraire (counter-clockwise operation) 9

Dans certains domaines aussi divers que l'océanographie, les machines-outils et la télévision, on relève les expressions à droite et à gauche, pour lesquelles il est sous-entendu que la rotation s'effectue dans la direction indiquée, à partir de la zone supérieure de l'axe. 
TOUCH A MARK, TO aborder une marque

TOUCHING A MARK abordage d'une marque

TRAPEZE HARNESS harnais de trapèze

TRIMMING OF SAILS réglage des voiles

UNDER WAY en route

UNSEEN MARK marque non aperçue

UNSHIPPING BALLAST débarquement du lest

WARNING SIGNAL (White shape, 10 minutes, one gun) signal d'attention

WATER! de l'eau!

WEIGHING OF WET CLOTHING pesée des vêtements mouillés

WINDWARD LEG partie (de parcours) montante ; bord montant

WINDWARD MARK marque au vent

WINDWARD SIDE côté au vent

WORKING SAIL voile de route, de louvoyage

YACHT ENTERED yacht inscrit

YACHT ENTITLED TO RACE yacht admis à courir

YACHT JIBING yacht empannant

YACHT MATERIALLY PREJUDICED yacht notablement lésé

YACHTS ON OPPOSITE TACKS yachts sur des bords opposés

YACHTS ON THE SAME TACK yachts sur le même bord

YACHTSMAN yachtman

YOU TACK! virez!

PIERRE BIron 